\title{
A metafluid with multistable density and internal energy states
}

Ofek Peretz ( $\nabla$ ofekperetz@campus.technion.ac.il)

Technion - Israel Institute of Technology https://orcid.org/0000-0002-4420-8706

\section{Ezra Ben Abu}

Technion - Israel institute of technology

\section{Anna Zigelman}

Technion - Israel Institute of Technology

\section{Sefi Givli}

Technion - Israel institute of technology

\section{Amir Gat}

Technion - Israel Institute of Technology https://orcid.org/0000-0001-8925-3001

\section{Article}

Keywords: artificial fluid, metafluid, stable states

Posted Date: July 9th, 2021

DOI: https://doi.org/10.21203/rs.3.rs-244708/v1

License: (c) (1) This work is licensed under a Creative Commons Attribution 4.0 International License. Read Full License

Version of Record: A version of this preprint was published at Nature Communications on April 5th, 2022. See the published version at https://doi.org/10.1038/s41467-022-29048-3. 


\title{
A metafluid with multistable density and internal energy states
}

\author{
Ofek Peretz ${ }^{1, *}$, Ezra Ben Abu ${ }^{1}$, Anna Zigelman ${ }^{1}$, Sefi Givli ${ }^{1}$, and Amir D. Gat ${ }^{1}$ \\ ${ }^{1}$ Faculty of Mechanical Engineering, Technion - Israel Institute of Technology, Haifa 3200003, Israel. \\ *ofekperetz@campus.technion.ac.il
}

\begin{abstract}
The efficiency, operation range, and environmental safety of energy and refrigeration cycles are determined by the thermodynamic properties of available fluids. We here suggest combining gas, liquid and multistable elastic capsules to create an artificial fluid with a multitude of stable states. We study, theoretically and experimentally, the suspension's internal energy, equilibrium pressure-density relations and their stability for both adiabatic and isothermal processes. We show that the elastic multistability of the capsules endows the fluid with multistable thermodynamic properties, including the ability of capturing and storing energy at standard atmospheric conditions, not found in naturally available fluids.
\end{abstract}

\section{Introduction}

The thermodynamic properties of fluids are crucial to many fields, and specifically to energy and refrigeration cycles $^{1,2}$. Creating a fluid with exceptional properties can thus be of great practical importance, yielding new possibilities of advancement. In this work we study the properties of a suspension composed of a multitude of lubricated multistable capsules (structures capable of transforming between different equilibrium deformation patterns $^{3,4}$ ) enclosing a compressible gas and immersed within another fluid. The thermodynamic properties of the suspension are determined by an interaction between the external liquid, the encapsulated gas, and the characteristic elastic energy profile of the capsules. By leveraging the elastic multistability of the suspended capsules, we can produce a fluid with multiple stable density points for a given pressure and temperature states as well as unstable regions.

Multistable structures are a special class of mechanical meta-materials. Meta-materials are architected structures made from assemblies of micro-level building blocks, usually arranged in a repeated pattern. While made from standard materials, the geometry of their building blocks can give rise to unique behaviors ${ }^{5}$. For example, metamaterials have been designed to have "negative mass" ${ }^{6-8}$, to manipulate light ${ }^{9,10}$ and stress waves ${ }^{11-13}$, or to provide ultra-high stiffness to weight ratio ${ }^{14}$. Multistable meta-materials feature a multitude of possible equilibrium configurations for a prescribed load. These meta-stable configurations differ in the state of each mechanical unit (building block), which leads to a highly inhomogeneous and non-affine deformation field for the whole body ${ }^{15}$. Hence, by careful design of their building blocks, these multi-stable structures can be "programmed" to undergo morphological changes in response to external stimuli. The ability to manufacture multi-stable meta-materials at all scales has opened exciting possibilities in a range of applications such as vibration isolation, super-elastic behavior, sensing and actuation, soft robotics, deployable structures and more ${ }^{16-25}$.

In this work we adopt a similar approach for the creation of multistable metafluids. We study the pressure-density relations, and stability, of a "metafluid", composed of a multitude of multistable gas-filled capsules suspended in a liquid. A simple example of a multistable capsule, used in the current study, is presented in Fig. 1, showing multiple connected bi-stable elements, produced by combining two non-identical conical frusta in opposing orientations. Bi-stability is therefore achieved by switching between extended and collapsed states through inversion of one frustum which creates a significant change in volume. Below, we fully define our model problem $(\S 2)$, discuss our approach for energy calculation ( $\$ 3)$, present our results for stable and unstable equilibria ( $(4)$, present experimental data of pressure cycles $(\S 5)$, and provide concluding remarks $(\S 6)$. 


\section{Problem definition}

We study the thermodynamic pressure-density relations and their stability for a fluidic suspension composed of multistable elastic capsules, enclosing a compressible gas and immersed in a liquid, as seen in Fig. 1. Each capsule is composed of $n$ identical bi-stable elastic elements. For the sake of simplicity, body forces are hereafter neglected. We examine a control volume which includes a large number of capsules, so that homogenization may be applied, while keeping all physical properties spatially uniform. The density of the external liquid is denoted by $\rho_{l}$. The number of capsules per-unit-mass is denoted by $\Phi$. The total mass of the capsules per suspension mass $m$ can be calculated via $\Phi$, as $m \Phi m_{c}$, where $m_{c}$ is the mass of a single capsule. We define $\Psi$ as the external fluid mass ratio. Thus, the mass of the external liquid is $m_{l}=\Psi m$ and the total mass of the capsules is $m(1-\Psi)$. Thus we obtain a $\Phi-\Psi$ relation of $\Phi=(1-\Psi) / m_{c}$.

Since the external fluid is assumed to be incompressible and thus its volume is constant, the equivalent density $\rho$ of the suspension can be expressed by

$$
\rho=\left[\frac{1-\Phi m_{c}}{\rho_{l}}+\Phi\left(v_{a}+\sum_{i=1}^{n} v_{i}\right)\right]^{-1}
$$

where $v_{i}$ is the gas-filled volume contained within bi-stable element $i$, and $v_{a}$ is all the additional gas and solid volume of the capsule.

The volume $v_{i}$ is a multi-valued function of the difference between the pressure of the internal gas, denoted as $p_{\text {gas }}$ and the pressure in the external fluid, denoted simply as $p$. We thus define this pressure difference by $p_{e l}=p_{g a s}-p$. We approximate $v_{i}$ by the tri-linear model (see Fig. 1B) which is a common and useful approximation that enables a simplified analytic treatment of bi-stability. More specifically, according to this model, when considering the energy relation to the pressure $p_{e l}$ and element volume $v_{i}$, the energy function may be approximated by three quadratic functionals ${ }^{15,26-30}$ leading to linear pressure-volume relation.

The tri-linear curve is defined by the stiffnesses $k_{\mathrm{I}}$ and $k_{\mathrm{II}}$, and the stability threshold points $\left(v_{s}^{\mathrm{I}}, p_{s}^{\text {open }}\right)$ and $\left(v_{s}^{\mathrm{II}}, p_{s}^{\text {close }}\right)$ in phases I and II, respectively (see definitions in Fig. 1B). Thus, the possible pressure range for bi-stable elements in phase I is $p_{e l}<p_{s}^{\text {open }}$, in the Spinodal region $p_{s}^{\text {close }}<p_{e l}<p_{s}^{\text {open }}$, and in phase II $p_{e l}>p_{s}^{\text {close }}$. These coefficients are commonly evaluated by fitting to an experiment ${ }^{20,31,32}$. Thus, the pressure-volume function for each of the bi-stable elements is given by,

$$
p_{e l}=\left\{\begin{array}{l}
p_{e l}^{\mathrm{I}} \\
p_{e l}^{s} \\
p_{e l}^{\mathrm{II}}
\end{array}\right\}=\left\{\begin{array}{c}
k_{\mathrm{I}}\left(v_{i}-v_{s}^{\mathrm{I}}\right)+p_{s}^{\text {open }} \\
k_{s}\left(v_{i}-v_{s}^{\mathrm{I}}\right)+p_{s}^{\text {open }} \\
k_{\mathrm{II}}\left(v_{i}-v_{s}^{\mathrm{II}}\right)+p_{s}^{\text {close }}
\end{array}\right\}, \begin{gathered}
v_{i}<v_{s}^{\mathrm{I}} \\
v_{s}^{\mathrm{I}}<v_{i}<v_{s}^{\mathrm{II}} \\
v_{s}^{\mathrm{II}}<v_{i}
\end{gathered}
$$

where $k_{s}$ is determined by requiring continuity between the spinodal region and I and II, yielding $k_{s}=\left(p_{s}^{\text {close }}-\right.$ $\left.p_{s}^{\text {open }}\right) /\left(v_{s}^{\mathrm{II}}-v_{s}^{\mathrm{I}}\right)<0$.

Next, we address the total gas-filled volume of a capsule, which is composed of the sum of all volumes of the $n$ bi-stable elements (given by $\sum_{i=1}^{n} v_{i}$ and additional gas-filled volume $v_{a, g a s}$ ). Since each element of the capsule can be in one of the three possible phases, the volume of a capsule is determined by the combination of all elements' states, denoted hereafter as the multistable state permutation $\overrightarrow{p e r}$. The permutation

$$
\overrightarrow{p e r}=\left\{n_{\mathrm{I}}, n_{s}, n_{\mathrm{II}}\right\}
$$

is defined by the number of elements in phase I $\left(n_{\mathrm{I}}\right)$, in the spinodal region $\left(n_{s}\right)$, and in phase II $\left(n_{\mathrm{II}}\right)$, where $n_{\mathrm{I}}+n_{s}+n_{\mathrm{II}}=n$. Thus, a capsule with 18 bistable elements (as in the presented experiments) has 190 possible permutations. Considering that the pressure acting on all elements is identical and equal to $p_{e l}$, we can calculate the volume of an element in each phase according to equation (2). Denoting the volume in each phase by $v_{\mathrm{I}}\left(p_{e l}\right), v_{\mathrm{s}}\left(p_{e l}\right), v_{\mathrm{II}}\left(p_{e l}\right)$, we obtain the total volume of gas within a capsule as $\overrightarrow{p e r} \cdot\left\{v_{\mathrm{I}}\left(p_{e l}\right), v_{\mathrm{s}}\left(p_{e l}\right), v_{\mathrm{II}}\left(p_{e l}\right)\right\}+v_{a, g a s}$. 

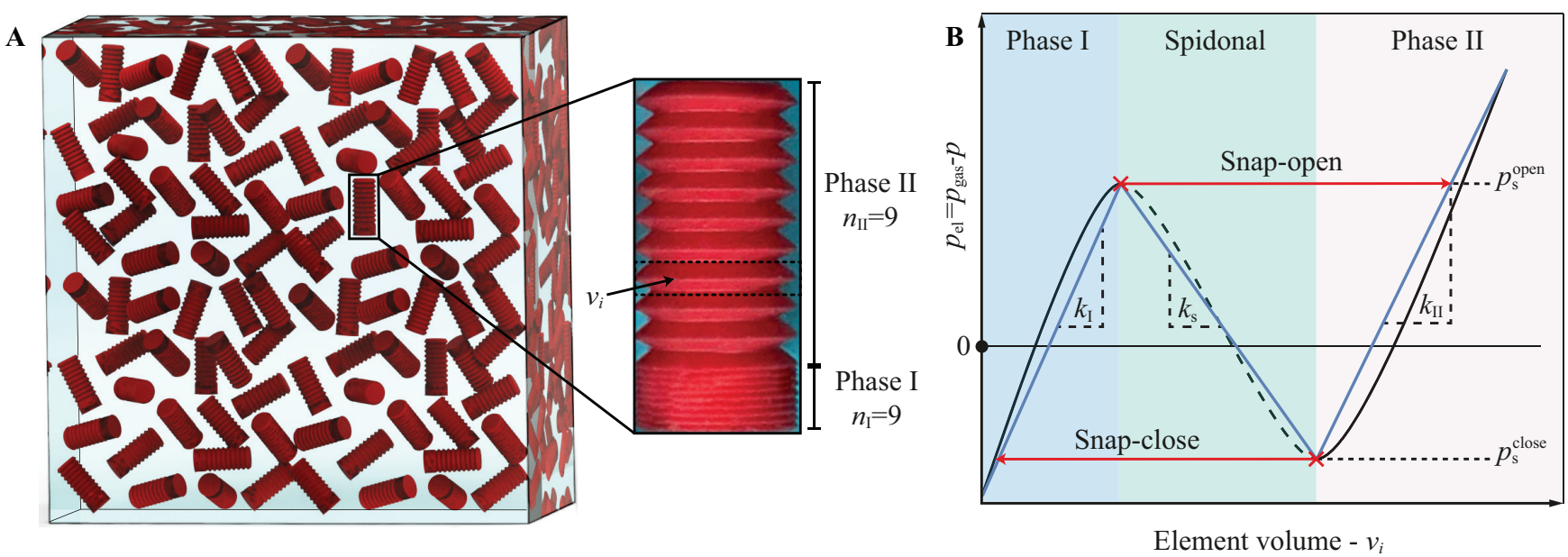

Figure 1. (A.) Schematic illustration of a "metafluid" containing encapsulated gas within multistable capsules, immersed with a second fluid. (B.) Pressure $\left(p_{e l}\right)$ vs. volume $\left(v_{i}\right)$ relation (shown by black continuous and dashed line) and the tri-linear approximation (shown by blue line) for each individual bi-stable element $(i)$ in the multi-stable capsule, where phase I, spinodal, and phase II regions are indicated.

\section{Internal energy}

We now calculate the internal energy for all of the possible permutations $\left\{n_{\mathrm{I}}, n_{s}, n_{\mathrm{II}}\right\}$ of the suspension. For the current analysis, we neglect the compressibility of the external fluid and assume that all capsules are identical. Thus, the internal energy of the suspension is composed of the total energy of the ideal gas, denoted by $U_{g a s}$, and the energy of the multistable elastic capsules, denoted by $U_{e l}$.

The elastic energy $U_{e l}$ of a bi-stable element $i$ for the three possible phases is given by integration of (2) over $v_{i}$, yielding

$$
\left\{\begin{array}{c}
U_{e l}^{\mathrm{I}} \\
U_{e l}^{s} \\
U_{e l}^{\mathrm{II}}
\end{array}\right\}=\left\{\begin{array}{cc}
k_{\mathrm{I}}\left(v_{i}-v_{s}^{\mathrm{I}}\right)^{2} / 2+p_{s}^{\text {open }} v_{i} \\
k_{s}\left(v_{i}-v_{s}^{\mathrm{I}}\right)^{2} / 2+p_{s}^{\text {open }} v_{i} \\
k_{\mathrm{II}}\left(v_{i}-v_{s}^{\mathrm{II}}\right)^{2} / 2+p_{s}^{\text {close }} v_{i}
\end{array}\right\}, \begin{gathered}
v_{i}<v_{s}^{\mathrm{I}} \\
v_{s}^{\mathrm{I}}<v_{i}<v_{s}^{\mathrm{II}} \\
v_{s}^{\mathrm{II}}<v_{i}
\end{gathered}
$$

and the total elastic potential energy per-unit-mass is thus

$$
U_{e l}=\Phi \cdot \overrightarrow{p e r} \cdot\left\{U_{e l}^{\mathrm{I}}, U_{e l}^{s}, U_{e l}^{\mathrm{II}}\right\} .
$$

To calculate $U_{\text {gas }}$ we assume an ideal gas model, $p_{\text {gas }}=\rho_{\text {gas }} R T$. For isothermal processes, we thus obtain the relation $p_{\text {gas }}=m_{g} R T /\left(v_{\text {agas }}+\overrightarrow{p e r} \cdot\left\{v_{\mathrm{I}}, v_{\mathrm{s}}, v_{\mathrm{II}}\right\}\right)$, where $R$ is the specific gas constant, $T$ is the constant temperature, $m_{g}$ is the mass of the gas within the capsule. For adiabatic processes, assuming ideal gas and isentropic compression/expansion, the gas pressure is given by $p_{\text {gas }} / p_{\text {atm }}=\left[v_{\text {atm }} /\left(v_{a, g a s}+\overrightarrow{p e r} \cdot\left\{v_{\mathrm{I}}, v_{\mathrm{s}}, v_{\mathrm{II}}\right\}\right)\right]^{\gamma}$, where $p_{\text {atm }}$ is atmospheric pressure, and $v_{a t m}$ is the volume taken by the gas contained within a single capsule at atmospheric pressure. In the adiabatic case, the temperature of the gas within the capsules will change during pressure variations according to $T=T_{0}\left(p / p_{0}\right)^{1-1 / \gamma}$, but not the temperature of the external incompressible fluid. These spatial temperature variations may yield heat transfer between the gas, solid and liquid, and more complex dynamics. To allow simple treatment of this case, we assume in the current analysis that an adiabatic process corresponds to the more strict requirement of no heat transfer from the encapsulated gas.

The gas energy per-unit-mass of the suspension is defined as

$$
U_{\text {gas }}=-\Phi \int_{v_{a t m}}^{v_{a, g a s}+\overrightarrow{p e r} \cdot\left\{v_{\mathrm{I}}, v_{\mathrm{s}}, v_{I I}\right\}} p_{g a s} d v
$$




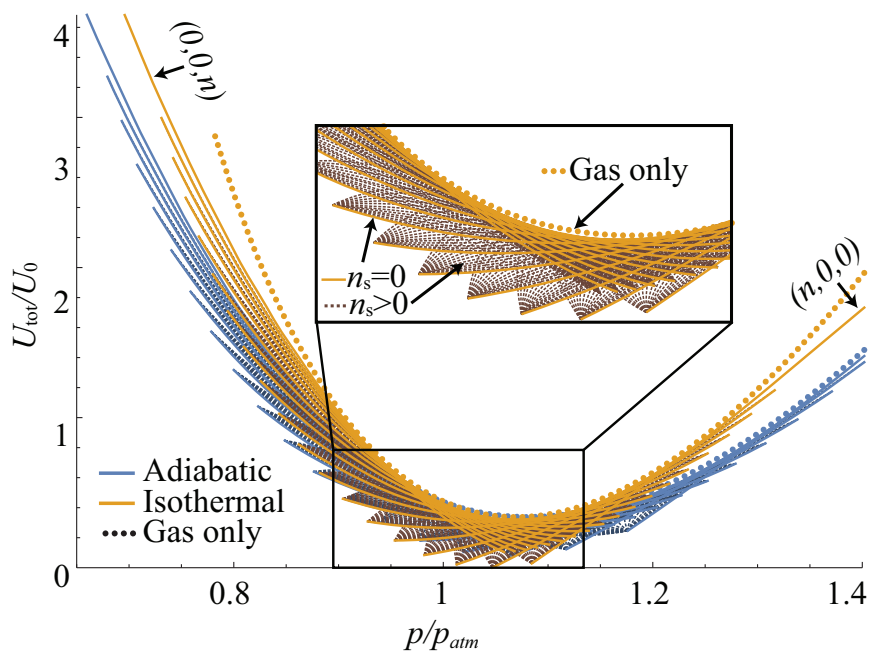

Figure 2. Internal energy per unit mass, $U_{\text {tot }} / U_{0}$, vs pressure, $p / p_{\text {atm }}$, where $U_{0}=\Phi p_{\text {atm }} v_{\text {atm }}$. All parameters were taken from experimental measurements and are defined below (see caption of Fig. 4). Adiabatic and isothermal cases are shown by blue and orange lines, respectively, where dashed lines denote states with bi-stable elements in the spinodal $\left(n_{s} \neq 0\right)$. Dotted curves of the corresponding colors represent the energy-pressure relation for an ideal gas. The multiple possible internal energies per external pressure allow to store, or release, energy at atmospheric conditions. The density of possible states decreases as the distance to the global minimum increases.

and thus the total energy of the system for an adiabatic process is given by $U_{t o t}=U_{g a s}+U_{e l}+U_{e x}$,

$$
\begin{array}{r}
U_{t o t}=\frac{\Phi p_{a t m} v_{a t m}}{\gamma-1}\left[\left(\frac{v_{a t m}}{v_{a, g a s}+\overrightarrow{p e r} \cdot\left\{v_{\mathrm{I}}, v_{\mathrm{s}}, v_{\mathrm{II}}\right\}}\right)^{\gamma-1}-1\right]+\Phi\left\{\begin{array}{c}
k_{\mathrm{I}}\left(v_{\mathrm{I}}-v_{s}^{\mathrm{I}}\right)^{2} / 2+p_{s}^{\text {open }} v_{\mathrm{I}} \\
k_{s}\left(v_{\mathrm{s}}-v_{s}^{\mathrm{I}}\right)^{2} / 2+p_{s}^{\text {open }} v_{\mathrm{s}} \\
k_{\mathrm{II}}\left(v_{\mathrm{II}}-v_{s}^{\mathrm{II}}\right)^{2} / 2+p_{s}^{\text {close }} v_{\mathrm{II}}
\end{array}\right\} \cdot \overrightarrow{p e r} \\
+\Phi p\left[v_{a, g a s}+\overrightarrow{p e r} \cdot\left\{v_{\mathrm{I}}, v_{\mathrm{s}}, v_{\mathrm{II}}\right\}-v_{a t m}\right]
\end{array}
$$

where the last RHS term is the external work energy $U_{e x}$ and $p$ is the dictated pressure in the external fluid. For an isothermal process the first RHS term is replaced with $\Phi p_{a t m} \ln \left[\left(v_{a, g a s}+\overrightarrow{p e r} \cdot\left\{v_{\mathrm{I}}, v_{\mathrm{s}}, v_{\mathrm{II}}\right\}\right) / v_{a t m}\right]$.

Equilibrium of the system occurs when the elastic force in all bistable elements is balanced by the difference between the external pressure, and the pressure of the internal gas. In the current analysis, all bi-stable elements are identical, and can be in one of three possible states (phase I, spinodal, and phase II). Thus, the equilibrium values in each of the three possible states can be calculated from the following relations,

$$
k_{\mathrm{I}}\left(v_{\mathrm{I}}-v_{s}^{\mathrm{I}}\right)+p_{s}^{\text {open }}=k_{s}\left(v_{\mathrm{s}}-v_{s}^{\mathrm{I}}\right)+p_{s}^{\text {open }}=k_{\mathrm{II}}\left(v_{\mathrm{II}}-v_{s}^{\mathrm{II}}\right)+p_{s}^{\text {close }}=p_{\text {atm }}\left[\frac{v_{\text {atm }}}{v_{a, g a s}+\overrightarrow{p e r} \cdot\left\{v_{\mathrm{I}}, v_{\mathrm{s}}, v_{\mathrm{II}}\right\}}\right]^{\gamma}-p .
$$

Equation (8) describes an adiabatic process, and for an isothermal process the last most RHS term is replaced with $\left(m_{g} R T\right) /\left(v_{a, g a s}+\overrightarrow{p e r} \cdot\left\{v_{\mathrm{I}}, v_{\mathrm{s}}, v_{\mathrm{II}}\right\}\right)$.

Solving equations (8) for the equilibrium values $v_{\mathrm{I}}, v_{\mathrm{S}}$ and $v_{\mathrm{II}}$, and substituting these into the energy equation (7) allows to calculate the energy of the suspension per given permutation $\overrightarrow{p e r}=\left\{n_{\mathrm{I}}, n_{s}, n_{\mathrm{II}}\right\}$ and external pressure $p$. Each permutation is only valid in a specific range of external pressures, as defined in equation (4). In Fig. 2 we present all of the available energy equilibria states, for multistable capsules composed of 18 bi-stable elements, with 190 possible permutations. Both isothermal (orange lines) and adiabatic (blue lines) processes are presented. The lines are determined by changing the external pressure, for different permutations of the phases of the bi-stable elements. The results show that the multi-stability extends the possible state of an ideal gas from a single line to a region containing multitude of possible energies. Different permutations provide different energy-pressure 
curves, corresponding to a fluid with different properties. Changing the properties of the fluid can be achieved by increasing/reducing the pressure beyond the range of a specific permutation. In addition to changing fluid properties, such transitions between different equilibrium states allow the fluid to store and release energy, as discussed below.

\section{Stability and the equation of state}

We examine the stability of equilibrium states by considering the Hessian of the total energy $U_{t o t}$, given by $H_{i j}=\partial^{2} U_{t o t} / \partial v_{i} \partial v_{j}$. An equilibrium configuration is stable if it corresponds to a local minimum of the energy, requiring positive definite Hessian. In order to obtain the Hessian in terms of all $n$ degrees of freedom, $v_{i}$, we note that $\overrightarrow{p e r} \cdot\left\{v_{\mathrm{I}}, v_{s}, v_{\mathrm{II}}\right\}=\sum_{j=1}^{n} v_{j}$. Substituting this relation into equation (7) and differentiating the total energy with respect to the degrees of freedom, $v_{i}$, yields the following set of $n$ equations

$$
\frac{\partial U_{t o t}}{\partial v_{i}}=p_{e l}^{\alpha(i)}\left(v_{i}\right)+\left(\frac{v_{a t m}}{v_{a, g a s}+\sum_{j=1}^{n} v_{j}}\right)-p=0, \quad i=1, \ldots, n,
$$

where $\alpha(i)$ denotes the phase of bi-stable element $i, \alpha(i) \in\{\mathrm{I}, s, \mathrm{II}\}$. The set of $n$ equations in (9) is differentiated again in order to obtain the Hessian matrix,

$$
\frac{\partial^{2} U_{t o t}}{\partial v_{i} \partial v_{j}}=\delta_{i j} k_{\alpha(i)}+k_{g}, \quad i, j=1, \ldots, n,
$$

where $k_{\alpha(i)}(i=1,2, \ldots, n)$ is the stiffness of the $i$-th bistable element and $k_{g}$ is the (instantaneous) stiffness of the gas at the equilibrium configuration. For an adiabatic process $k_{g}=\left(\gamma p_{\text {atm }} / v_{\text {atm }}\right)\left[v_{\text {atm }} /\left(v_{a, g a s}+\overrightarrow{p e r} \cdot\left\{v_{\mathrm{I}}, v_{\mathrm{s}}, v_{\mathrm{II}}\right\}\right)\right]^{\gamma+1}$ and for isothermal process $k_{g}=v_{\text {atm }} p_{\text {atm }} /\left(v_{a, g a s}+\overrightarrow{p e r} \cdot\left\{v_{\mathrm{I}}, v_{\mathrm{s}}, v_{\mathrm{II}}\right\}\right)^{2}$.

An equilibrium state is stable if and only if the Hessian is positive definite, which occurs if and only if all leading principal minors $A_{j}$ (the determinant of the submatrix of first $j$ rows and $j$ columns) are positive. The principal minors of the current configuration in (10) are

$$
A_{j}=\left(\prod_{i=1}^{j} k_{\alpha(i)}\right)\left[1+k_{g} \sum_{i=1}^{j} \frac{1}{k_{\alpha(i)}}\right] .
$$

In order to obtain conditions for positive $A_{j}$, we examine separately three possible permutation states. In the first state the permutation is of the form $\left(n_{\mathrm{I}}, 0, n_{\mathrm{II}}\right)$ and thus there are no elements in the spinodal region. In this case, since all $k_{\alpha(i)}$ and $k_{g}$ are positive, all minors $A_{j}$ in (11) are necessarily positive for all $p$. Another possible permutation state is the case of two or more elements in the spinodal region. The symmetry in terms of the degrees of freedom allows us to arbitrarily rearrange the degrees of freedom. We re-order the Hessian so that the first two elements are in the spinodal region, and thus $k_{\alpha(1)}=k_{\alpha(2)}=k_{s}<0$. The first minor in this case is given by $A_{1}=k_{g} k_{s}\left(1 / k_{g}+1 / k_{s}\right)$ and is positive only if $k_{g}>\left|k_{s}\right|$. The second minor $\left.A_{2}=k_{g}\left(k_{s}\right)^{2}\left(1 / k_{g}+2 / k_{s}\right)\right)$ is positive only if $k_{g}<\left|k_{s}\right| / 2$. Thus, since the two conditions cannot hold simultaneously, if there are at least two elements in the spinodal region, the configuration is unstable for all $k_{g}$. Finally, we examine the case of permutations of the form $\left(n_{\mathrm{I}}, 1, n_{\mathrm{II}}\right)$, meaning that only a single element is in the spinodal region. We re-order the Hessian so that the $n$-th element is in the spinodal region. Thus all minors $A_{j}, j \in\{1, \ldots, n-1\}$, are positive. The requirement that the minor $A_{n}$ is positive, which is needed for the stability condition to be satisfied, may be expressed for an adiabatic process as

$$
\frac{v_{a t m}}{\gamma p_{a t m}}\left(\frac{v_{a, g a s}+\overrightarrow{p e r} \cdot\left\{v_{\mathrm{I}}, v_{\mathrm{s}}, v_{\mathrm{II}}\right\}}{v_{a t m}}\right)^{\gamma+1}+\overrightarrow{p e r} \cdot\left\{\frac{1}{k_{\mathrm{I}}}, \frac{1}{k_{\mathrm{S}}}, \frac{1}{k_{\mathrm{II}}}\right\}<0
$$

where $\left\{v_{\mathrm{I}}, v_{\mathrm{s}}, v_{\mathrm{II}}\right\}$ are functions of the external pressure $p$, and are calculated from equation (8). For isothermal process the first LHS term is replaced with $\left(v_{a, g a s}+\overrightarrow{p e r} \cdot\left\{v_{\mathrm{I}}, v_{\mathrm{s}}, v_{\mathrm{II}}\right\}\right)^{2} /\left(v_{\text {atm }} p_{a t m}\right)$. Obtaining the stability condition 

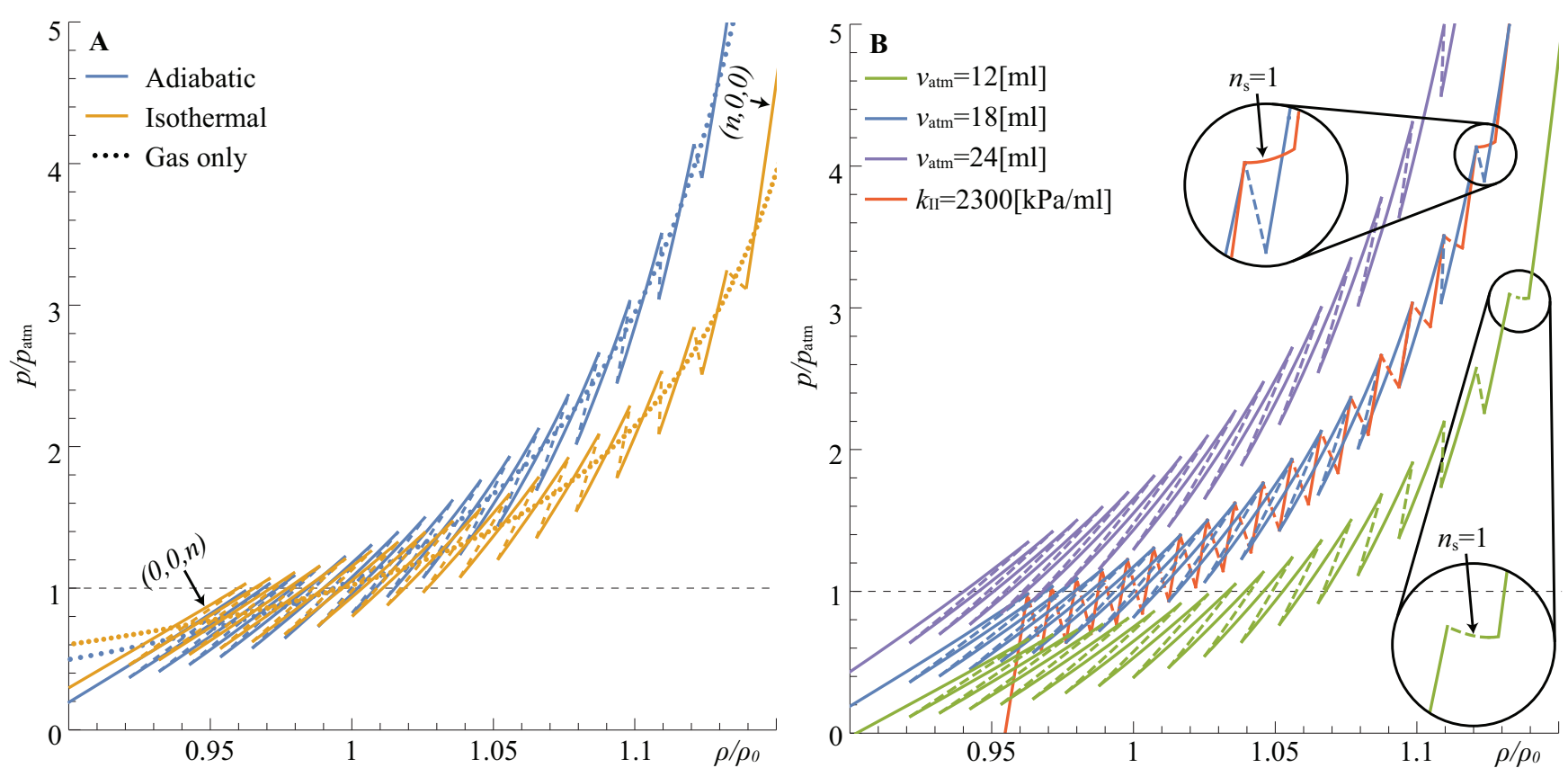

Figure 3. Pressure ( $\left.p / p_{\text {atm }}\right)$ vs. density $\left(\rho / \rho_{0}\right)$ for stable (solid lines) and unstable (dashed lines) equilibria states. The physical parameters used are listed below (see caption of Fig. 4). (A.) Comparison between adiabatic (blue lines) and isothermal (orange lines) processes. Dotted lines represent ideal gas. (B.) Examining the effect of $v_{a t m}$, the volume of the gas encapsulated in a single capsule at atmospheric pressure, and $k_{\mathrm{II}}$, the slope of phase II. The blue line is a reference curve based on experimental values, with $v_{a t m}=18 \mathrm{ml}$ and $k_{\mathrm{II}}=152 \mathrm{kPa} / \mathrm{ml}$. The green and purple lines are pressure-density curves for fluid with decreased and increased values of $v_{\text {atm }}$ of $12 \mathrm{ml}$ and $24 \mathrm{ml}$, respectively. The red line is a pressure-density curve for a fluid with positive slope at unstable regions, achieved by setting $k_{\mathrm{II}}=2300 \mathrm{kPa} / \mathrm{ml}$, while keeping all other parameters identical to the blue reference curve. The insets show that decreasing $v_{\text {atm }}$ or increasing $k_{\mathrm{II}}$ creates regions where the fluid is stable although a bi-stable element is at the spinodal region.

allows us to present all possible density-pressure relations which correspond to all permutations of the form $\overrightarrow{p e r}=\left(n_{\mathrm{I}}, 0, n_{\mathrm{II}}\right)$ or permutations of the form $\overrightarrow{p e r}=\left(n_{\mathrm{I}}, 1, n_{\mathrm{II}}\right)$ along with the condition $(12)$.

The pressure-density relations of the fluid are presented in Fig. 3, where unstable states are marked by dashed lines and stable states are marked by solid lines. In Fig. 3A we present the external pressure $p$ vs density $\rho$ for adiabatic (blue lines) and isothermal (orange lines) processes. In addition, pressure-density relations for gas only are presented by dotted lines with corresponding colors. The distance between stable points of the fluid is minimal near the permutation $\overrightarrow{p e r}=(0,0, n)$ and increases as we approach the reversed order permutation $\overrightarrow{p e r}=(n, 0,0)$. In Fig. 2B, we show adiabatic processes, which were obtained for three different initial gas volumes within the capsules at atmospheric conditions (blue line is the reference curve, purple line has decreased amount of gas and green have increased amount of gas). The effect of the amount of gas becomes more pronounced as the density increases and the average slope of the multi-stable curve increases with the addition of gas into the capsules. In addition, it is possible to see that permutations of the form $\overrightarrow{p e r}=\left(n_{\mathrm{I}}, 1, n_{\mathrm{II}}\right)$ become stable as the density of the fluid increases (see insets within Fig. 2B). The red curve in Fig. 2B, presents modified $k_{\mathrm{II}}$. In this case, a permutation with unstable element $\left(n_{s}=1\right)$ results in a stable state of the system. The insets show that decreasing $v_{a t m}$ or increasing $k_{\mathrm{II}}$ creates regions where the fluid is stable although a bi-stable element is at the spinodal region. In both panels, several stable densities are possible for a given pressure, as well as several pressures for a single density. This feature appears for unstable curves with positive slopes. 

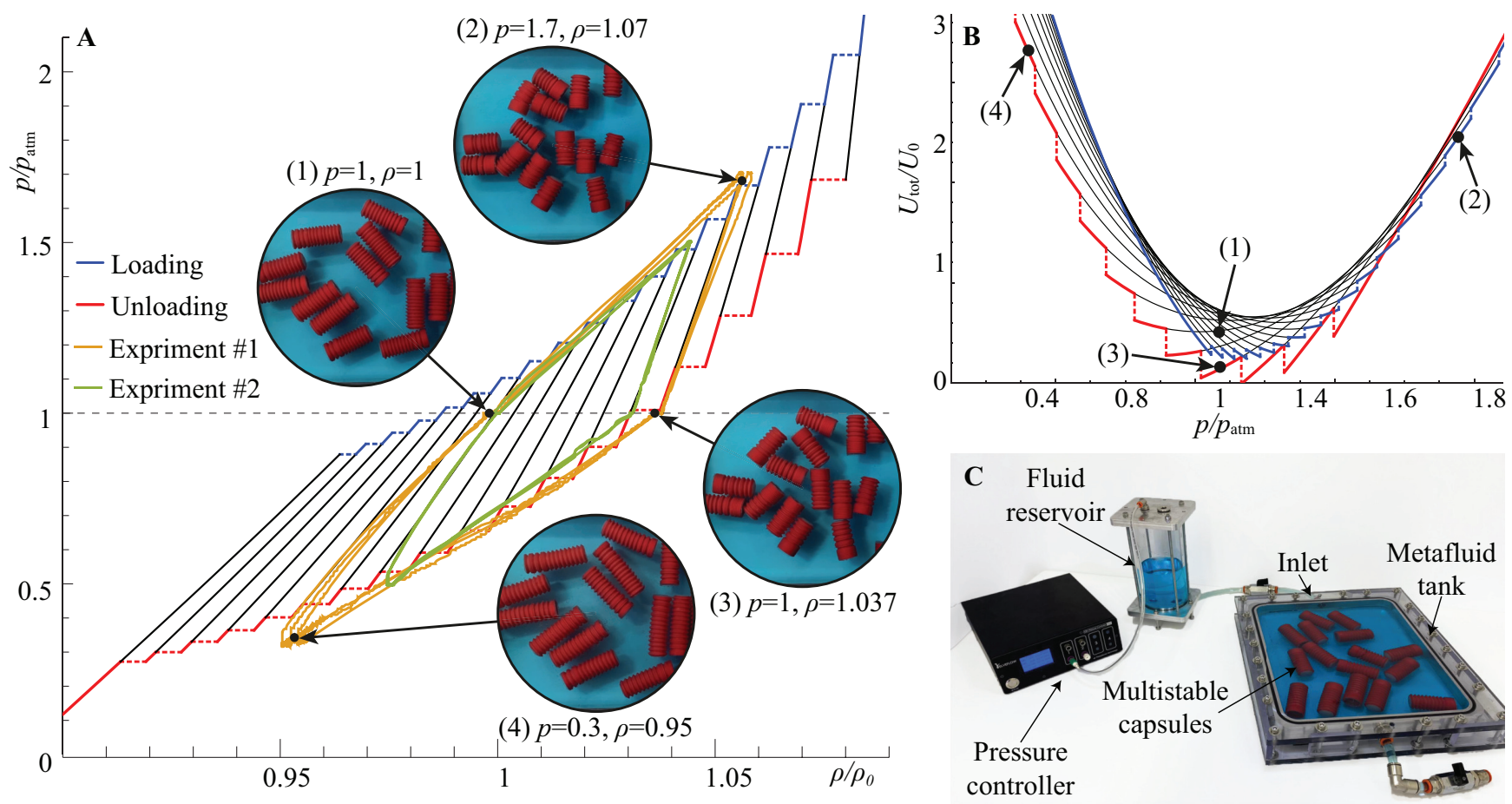

Figure 4. (A.) Experimental and theoretical pressure $\left(p / p_{\text {atm }}\right)$ vs. density $\left(\rho / \rho_{0}\right)$ cycles. The black, blue, and red curves represent (analytically calculated) equi-permutation lines, loading, and unloading modes, respectively. Orange and green curves represent the experimental results (each curve containing three cycles). Representative frames (circular insets) taken from our experiment correspond to points (1)-(4) on the orange curve. In the experimental data, snap-open and snap-close points are $\left(v_{s}^{\mathrm{I}}, p_{s}^{\text {open }}\right)=(0.25 \mathrm{ml}, 26.24 \mathrm{kPa})$ and $\left(v_{s}^{\mathrm{II}}, p_{s}^{\text {close }}\right)=(1.04 \mathrm{ml}$, $-16.61 \mathrm{kPa}$ ), respectively. The slopes of the tri-linear approximation are $k_{\mathrm{I}}=2300 \mathrm{kPa} / \mathrm{ml}, k_{\mathrm{II}}=152 \mathrm{kPa} / \mathrm{ml}$, for phase I and II, respectively, and the number of bi-stable elements per capsule is 18. (B.) The corresponding internal energy of the system, where points (1)-(4) correspond to the frames in (A). (C.) Experimental setup: the pressure controller, which is responsible for regulating the (constant) pressure in the fluid reservoir, is connected to the metafluid tank inlet via a flow valve. The height of the fluid in the fluid reservoir is monitored in the experiment to allow measuring the change in equivalent density of the metafluid. (SI - Movies) 


\section{Pressure cycles and experimental demonstration}

In Fig. 4 we present analytic and experimental results for cyclical pressurization of the fluid. In panel 4A, each of the black curves denotes a stable equilibrium state corresponding to a different permutation, while the blue and red curves follow the actual loading and unloading paths, respectively, involving switching between different permutations. In order to access one of the multiple possible densities per a given pressure, we need to reach the specific permutation on which the desired density-pressure point is located. This can be achieved by increasing the pressure beyond the initial snap-close value associated with the current permutation (blue line in Fig. 4A), until reaching a stable permutation for the given pressure value. At this point, reducing the pressure will not change the permutation as long as the pressure is above the snap-open value of the current permutation (red line in Fig. $4 \mathrm{~A}$ ) and the system will remain at the same permutation (following the black line in Fig. 4A associated with that permutation). Thus, multi-stable pressure cycles are closed curves which involve intervals on constant permutation curves (black lines), intervals on the snap-close curves (blue lines), and intervals on the snap-open curves (red lines). This unique ability to choose a specific operation curve is demonstrated experimentally in what follows.

The experimental setup (Fig 4C) consists of a cubic $35 \times 30 \times 3.2 \mathrm{~cm}$ fluid-filled tank, a pressure flow controller,

and a fluid reservoir. The capsules, illustrated in Fig 1, yield the following physical parameters: $v_{s}^{\mathrm{I}}=0.51 \mathrm{ml}$, $v_{s}^{\mathrm{II}}=1.04 \mathrm{ml}, k_{\mathrm{I}}=2300 \mathrm{kPa} / \mathrm{ml}, k_{\mathrm{II}}=152 \mathrm{kPa} / \mathrm{ml}$ and are composed of $n=18 \mathrm{bi}$-stable elements. The surrounding fluid is water $\left(\rho_{l}=0.997 \mathrm{~g} / \mathrm{ml}\right)$ and the internal fluid is air $(\gamma=1.4, R=0.286 \mathrm{~J} /(\mathrm{g} \mathrm{K}))$. The fluid container is connected to a pressure controller (Elveflow OB1), which is connected to the tank. Using this setup, we experimentally measured the snapping pressures (see definitions in Fig. 1) as $p_{s}^{\text {open }}=26.24 \mathrm{kPa}$ for the snap-open value and $p_{s}^{\text {close }}=-16.61$ $\mathrm{kPa}$ for the snap-close value, with standard deviation of $\pm 0.1 \mathrm{kPa}$ for both values. The measurements were based on averaging 6 experiments, where we used air actuation to eliminate transient fluidic effects. To obtain the change in density we measured the height of the fluid in the reservoir which is equivalent to the change in the volume of the capsules (since the surrounding fluid is assumed incompressible). Throughout the experiment, the height of the liquid in the fluid reservoir and the pressure in the tank were continuously monitored. By using the density of the surrounding fluid and the volume of the metafluid tank we calculated the equivalent density as a function of the pressure. The increase of tank volume due to elasticity of the walls was accounted for by pressurizing the tank without capsules, and measuring the volume-pressure relation.

In Fig. 4A, the orange and green curves present experimental results of two different pressure-density cycles of a multi-stable fluid. Each experimental curve presents three identical pressure cycles, repeated in each experiment. In Experiment \#1, the initial state of the capsules at atmospheric conditions yields equivalent density of $\rho_{0}=0.719$ $\mathrm{g} / \mathrm{ml}$ and permutation of $\overrightarrow{p e r}_{0}=\{5,0,13\}$ (see subpanel $\mathrm{A}(1)$ ). We pressurized the tank linearly from $p_{0}=101 \mathrm{kPa}$ to $171 \mathrm{kPa}$ over a period of 30 seconds. The fluid density increased and the permutations changed until reaching a steady state permutation of $\overrightarrow{p e r}=\{14,0,4\}$ and density of $\rho=1.07 \rho_{0}$ (see subpanel A(2)). At this stage, we reduced the pressure back to $p_{0}$ over a period of 30 seconds. In agreement with the model, the permutation of the fluid remained nearly unchanged $\left(\overrightarrow{p e r}=\{13,0,5\}\right.$ ) while the density decreased to $\rho=1.037 \rho_{0}$ (see subpanel $\mathrm{A}(3)$ ). To return to the initial state, we applied a pressure of $30 \mathrm{kPa}$ which allowed us to change the state back to the initial fluid permutation of $\overrightarrow{p e r}=\{5,0,13\}$ and the density of $\rho=0.95 \rho_{0}$ (see subpanel A(4)). Finally, increasing the pressure back to $101 \mathrm{kPa}$ compressed the fluid along a constant permutation. Similarly, in Experiment \#2 we applied a smaller cycle, in which points 1-4 have the densities of $\rho=\rho_{0}, \rho=1.04 \rho_{0}, \rho=0.97 \rho_{0}, \rho=1.03 \rho_{0}$ and the permutations of $\{7,0,11\},\{11,0,7\},\{11,0,7\},\{7,0,11\}$, respectively. As it is evident from Fig. 4, a good agreement is observed between the theoretic stability states of the system and the experimental results for both cycles (see SI-Movies).

In panel 4B we present the internal energy associated with the different equilibrium states. The internal energy stored within the fluid at atmospheric conditions in the initial state, point 1 , is released by adding sufficient energy to the fluid reaching a different permutation, point 2. From point 2, energy is released along a single permutation until reaching point 3 , which is the minimal energy at the atmospheric conditions. Similarly, storing energy within the fluid can be achieved by temporarily adding energy to change permutations, and reaching point 4 . Reducing the pressure back to atmospheric pressure will not change the fluid permutation, and some of the energy will thus 
remain stored within the fluid.

\section{Concluding remarks}

In this study, we introduced a new concept for a multistable metafluid, and investigated theoretically and experimentally the relation between pressure and density, for both adiabatic and isothermal processes. We showed that the multitude of stable density and energy states make it possible to capture and store energy at standard atmospheric conditions, which is not possible with naturally available fluids.

The experiments presented in this work involved large $O(\mathrm{~cm})$ particles. While these experiments aimed at demonstrating the concept, miniaturization of the multistable particles is, evidently, needed for creating metafluids for real applications. The results of our experiments suggest that the concept of multistable metafluids paves the way for the creation of futuristic non-polluting fluids with enhanced properties for energy and cooling cycles, leveraging multi-stability to harvest, store and release energy and heat.

The code used in the work are available in SI Appendix Code

\section{References}

1. Chen, H., Goswami, D. Y. \& Stefanakos, E. K. A review of thermodynamic cycles and working fluids for the conversion of low-grade heat. Renew. Sustain. Energy Rev. 14, 3059-3067 (2010).

2. Becker, T. M. et al. Absorption refrigeration cycles with ammonia-ionic liquid working pairs studied by molecular simulation. Ind. Eng. Chem. Res. 57, 5442-5452 (2018).

3. Peretz, O., Mishra, A. K., Shepherd, R. F. \& Gat, A. D. Underactuated fluidic control of a continuous multistable membrane. PNAS 117, 5217-5221 (2020).

4. Guest, S. D. \& Pellegrino, S. Analytical models for bistable cylindrical shells. Proc. R. Soc. A 462, 839-854 (2006).

5. Lee, J. H., Singer, J. P. \& Thomas, E. Micro-nanostructured mechanical metamaterials. Adv. Mater. 24, 4782-4810 (2012).

6. Correa, D. M. et al. Negative stiffness honeycombs for recoverable shock isolation. Rapid Prototyp. J. 21, 193-200 (2015).

7. Correa, D. M., Seepersad, C. C. \& Haberman, M. Mechanical design of negative stiffness honeycomb materials. Integrating Mater. Manuf. Innov. 4, 10 (2015).

8. Babaee, S. et al. 3d soft metamaterials with negative poisson's ratio. Rapid Prototyp. J. 25, 5044-5049 (2013).

9. Arpin, K. A. et al. Multidimensional architectures for functional optical devices. Adv. Mater. 22, 1084-1101 (2010).

10. Soukoulis, C. M. \& Wegener, M. Past achievements and future challenges in the development of threedimensional photonic metamaterials. Nat. Photonics 5, 523-530 (2011).

11. Peng, P. et al. Acoustic tunneling through artificial structures: From phononic crystals to acoustic metamaterials. Solid State Commun. 151, 400-403 (2011).

12. Lu, M. H., Feng, L. \& Chen, Y. F. Phononic crystals and acoustic metamaterials. Mater. Today 12, 34-42 (2009).

13. Deymier, P. Acoustic metamaterials and phononic crystals, in springer series in solid-state sciences. Springer Berlin Heidelberg : Imprint: Springer,: Berlin, Heidelberg. (2013).

14. Zheng, X. et al. Ultralight, ultrastiff mechanical metamaterials. Science 344, 1373-1377 (2014).

15. Puglisi, G. \& Truskinovsky, L. Mechanics of a discrete chain with bi-stable elements. J. Mech. Phys. Solids 48, $1-27$ (2000). 
16. Frenzel, T., Findeisen, C., Kadic, M., Gumbsch, P. \& Wegener, M. Tailored buckling microlattices as reusable light-weight shock absorbers. Adv. Mater. 28, 5865-5870 (2016).

17. Ha, C. S., Lakes, R. S. \& Plesha, M. E. Design, fabrication, and analysis of lattice exhibiting energy absorption via snap-through behavior. Mater. Des. 141, 426-437 (2018).

18. Leelavanichkul, S., Chekarev, A., Adams, D. O. \& Solzbacher, F. Energy absorption of a helicoidal bistable structure. J. Mech. Mater. Struct. 5, 305-321 (2010).

19. Yang, H. \& Ma, L. Multi-stable mechanical metamaterials by elastic buckling instability. J. Mater. Sci. 54, 3509-3526 (2019).

20. Cohen, T. \& Givli, S. Dynamics of a discrete chain of bi-stable elements: a biomimetic shock absorbing mechanism. J. Mech. Phys. Solids 64, 426-439 (2014).

21. Katz, S. \& Givli, S. Boomerons in a 1-d lattice with only nearest-neighbor interactions. EPL 131, 64002 (2020).

22. Shan, S. et al. Multistable architected materials for trapping elastic strain energy. Adv. Mater. 27, 4296-4301 (2015).

23. Rafsanjani, A., Akbarzadeh, A. \& Pasini, D. Snapping mechanical metamaterials under tension. Adv. Mater. 27, 5931-5935 (2015).

24. Pan, F. et al. 3d pixel mechanical metamaterials. Adv. Mater. 31, 1900548 (2019).

25. James, K. \& Waisman, H. Layout design of a bi-stable cardiovascular stent using topology optimization. Comput. Methods Appl. Mech. Eng. 305, 869-890 (2016).

26. Müller, I. \& Xu, H. On the pseudo-elastic hysteresis. Acta metallurgica et materialia 39, 263-271 (1991).

27. Fedelich, B. \& Zanzotto, G. Hysteresis in discrete systems of possibly interacting elements with a double-well energy. J. Nonlinear Sci. 2, 319-342 (1992).

28. Allinger, T. L., Epstein, M. \& Herzog, W. Stability of muscle fibers on the descending limb of the force-length relation. a theoretical consideration. J. biomechanics 29, 627-633 (1996).

29. Müller, I. \& Villaggio, P. A model for an elastic-plastic body. Arch. for Ration. Mech. Analysis 65, 25-46 (1977).

30. Shaw, J. A. \& Kyriakides, S. On the nucleation and propagation of phase transformation fronts in a niti alloy. Acta materialia 45, 683-700 (1997).

31. Luongo, A., Casciati, S. \& Zulli, D. Perturbation method for the dynamic analysis of a bistable oscillator under slow harmonic excitation. Smart structures systems 18, 183-196 (2016).

32. Benichou, I., Faran, E., Shilo, D. \& Givli, S. Application of a bi-stable chain model for the analysis of jerky twin boundary motion in nimnga. Appl. Phys. Lett. 102, 011912 (2013). 


\section{Supplementary Files}

This is a list of supplementary files associated with this preprint. Click to download.

- SIMovieX2.mp4

- SIMovieX1.mp4 